\title{
Phase diagram of van der Waals-like phase separation in a driven granular gas
}

\author{
Evgeniy Khain ${ }^{1}$, Baruch Meerson ${ }^{1}$, and Pavel V. Sasorov ${ }^{2}$ \\ ${ }^{1}$ Racah Institute of Physics, Hebrew University of Jerusalem, Jerusalem 91904, Israel and \\ ${ }^{2}$ Institute of Theoretical and Experimental Physics, Moscow 117218, Russia
}

\begin{abstract}
Equations of granular hydrostatics are used to compute the phase diagram of the recently discovered van der Waals-like phase separation in a driven granular gas. The model two-dimensional system consists of smooth hard disks in a rectangular box, colliding inelastically with each other and driven by a "thermal" wall at zero gravity. The spinodal line and the critical point of the phase separation are determined. Close to the critical point the spinodal and binodal (coexistence) lines are determined analytically. Effects of finite size of the confining box in the direction parallel to the thermal wall are investigated. These include suppression of the phase separation by heat conduction in the lateral direction and a change from supercritical to subcritical bifurcation.
\end{abstract}

PACS numbers: 45.70.Qj

\section{INTRODUCTION}

Granular gases (gases of inelastically colliding macroscopic particles) exhibit a plethora of symmetry-breaking instabilities and clustering phenomena [1]. Their investigation is useful both for testing and improving the models of granular flow, and for a deeper understanding of farfrom-equilibrium dynamics in general. In this work we focus our attention on a recently discovered phase separation instability which occurs in a prototypical twodimensional granular system: an assembly of monodisperse hard disks in a box, colliding inelastically with each other and driven, at zero gravity, by a rapidly vibrating or thermal wall. An immediate consequence of the inelasticity of the particle collisions is the formation of a laterally uniform cluster (the stripe state) at the wall opposite to the driving wall [2, 3]. Both granular hydrodynamics and direct molecular dynamic simulations show that this simple clustering state can exhibit spontaneous symmetry-breaking instability leading to phase separation: coexistence of dense and dilute regions of the granulate (droplets and bubbles) along the wall opposite to the driving wall $[4,5,6,67,8,[9,10]$. This far-fromequilibrium phase separation is strikingly similar to the gas-liquid transition in the classical van der Waals model. The objective of this work is a systematic investigation of the steady states in this system and computation of the phase diagram starting from the Navier-Stokes granular hydrodynamics. Granular hydrodynamics is expected to be an accurate leading order theory when the mean free path of the particles is much less than a characteristic inhomogeneity scale of the problem, and the mean time between two consecutive collisions of a particle is much less than any time scale the hydrodynamics attempts to describe. In addition, we should work with sufficiently low particle densities, when an account of binary collisions and volume exclusion effects is sufficient 11. As will be shown below (see also Ref. 2]), the requirement that the mean free path be small compared to the inhomogeneity scale implies in this system that particle collisions must be nearly elastic.
For steady states with a zero mean flow, granular hydrodynamic equations reduce to granular hydrostatics, see Section 2. The hydrostatic problem is fully described by three scaled parameters introduced below: the area fraction of the particles $f$, the aspect ratio of the box $\Delta$ and the effective hydrodynamic inelastic heat loss parameter $\Lambda$ [4, 6]. By solving the hydrostatic equations numerically, we obtain, in Section 2, the spinodal line and the critical point of the phase separation in the limit of $\Delta \rightarrow \infty$. Section 3 deals with the same limit of $\Delta \rightarrow \infty$ but, in addition, assumes a close proximity to the critical point. Here we find the spinodal and binodal (coexistence) lines analytically and determine the structure of the steady-state domain wall, separating the more dense and less dense stripes in the lateral direction. Effects of finite aspect ratio $\Delta$ are addressed in Sec. 4. These include suppression of the phase separation by heat conduction in the lateral direction and a change from a supercritical to subcritical bifurcation. Section 5 includes a brief discussion of the results and proposes directions of future work.

\section{HYDROSTATICS OF PHASE SEPARATION: MODEL AND GENERAL RESULTS}

In this Section we formulate the model, briefly review the stripe state and compute the spinodal line and the critical point of the phase separation for a laterally infinite system, $\Delta \rightarrow \infty$.

\section{A. Model}

Consider an assembly of inelastically colliding hard disks of diameter $d$ and mass $m=1$, moving in a box with dimensions $L_{x} \times L_{y}$ at zero gravity. Collisions of disks with the walls $x=0$ and $y= \pm L_{y} / 2$ are assumed elastic. Alternatively, periodic boundary conditions in the $y$-direction can be imposed. We refer to the $y$-direction as the lateral direction of the system. In a laterally infinite system $L_{y} \rightarrow \infty$. The particles are driven by a 
"thermal" wall: a constant granular temperature $T_{0}$ is prescribed at $x=L_{x}$. The inelasticity of the particle collisions is parametrized by a constant normal restitution coefficient $r$; we will work in the nearly elastic limit $1-r^{2} \ll 1$. We also assume a moderate number density $n: n / n_{c}<0.5$, where $n_{c}=2 /\left(\sqrt{3} d^{2}\right)$ is the hexagonal close packing density. The last two assumptions allow us to employ the Navier-Stokes granular hydrodynamics. Throughout this work, we will use for concrete calculations the constitutive relations suggested by Jenkins and Richman [1]. These relations were derived by analogy with those obtained in the framework of a successful but still empiric Enskog theory 12]. Detailed comparisons with molecular dynamic simulations shows that the error margin of the Enskog heat conductivity can reach as much as $10-15$ percent 13 . Still, these relations seem to be the best available constitutive relations for moderate densities. Importantly, most of the analytical results in this work are written in a more general form which only assumes a Navier-Stokes structure of hydrodynamic equations. The Jenkins-Richman's relations are used only for computing numerical factors.

Energy input at the thermal wall balances the energy loss due to inter-particle collisions, so we assume that the system reaches a steady state with a zero mean flow. Therefore, the full hydrodynamic equations reduce to hydrostatic equations:

$$
p=\text { const and } \nabla \cdot(\kappa \nabla T)=I
$$

where $p$ is the granular pressure, $T$ is the granular temperature, $\kappa$ is the thermal conductivity, and $I$ is the rate of energy loss by collisions. Notice that we did not account, in the second of Eq. (1), for an additional (inelastic) contribution to the heat flux which is proportional to the density gradient. For a dilute gas this term was derived in Ref. [14]. It can be neglected in the nearly elastic limit which is assumed throughout this paper.

The constitutive relations entering Eqs. (1) include the equation of state $p=p(n, T)$ and expressions for $\kappa$ and $I$ in terms of $n$ and $T$. In our notation, these relations can be written as 11$]$

$$
\begin{aligned}
p & =n T\left(1+2 G^{\prime}\right) \\
\kappa & =\frac{2 d n T^{1 / 2} G^{\prime}}{\sqrt{\pi}}\left[1+\frac{9 \pi}{16}\left(1+\frac{2}{3 G^{\prime}}\right)^{2}\right], \\
I & =\frac{8(1-r) n T^{3 / 2} G^{\prime}}{\sqrt{\pi} d} \\
G^{\prime} & =\frac{\nu\left(1-\frac{7 \nu}{16}\right)}{(1-\nu)^{2}}
\end{aligned}
$$

where $\nu=n\left(\pi d^{2} / 4\right)$ is the solid fraction. Let us rescale the coordinates by $L_{x}: \mathbf{r} / L_{x} \rightarrow \mathbf{r}$. In the rescaled coordinates the box dimensions become $1 \times \Delta$, where $\Delta=L_{y} / L_{x}$ is the aspect ratio of the box. Introducing a normalized inverse density $z(x, y)=n_{c} / n(x, y)$ and eliminating the temperature, one can rewrite Eqs. (1) as a single equation for $z(x, y)[2,4,6]$ :

$$
\nabla \cdot(F(z) \nabla z)=\Lambda Q(z)
$$

where $F(z)=A(z) B(z)$,

$$
\begin{aligned}
& A(z)=\frac{G\left[1+\frac{9 \pi}{16}\left(1+\frac{2}{3 G}\right)^{2}\right]}{z^{1 / 2}(1+2 G)^{5 / 2}} \\
& B(z)=1+2 G+\frac{\pi}{\sqrt{3}} \frac{z\left(z+\frac{\pi}{16 \sqrt{3}}\right)}{\left(z-\frac{\pi}{2 \sqrt{3}}\right)^{3}} \\
& Q(z)=\frac{6}{\pi} \frac{z^{1 / 2} G}{(1+2 G)^{3 / 2}} \\
& G(z)=\frac{\pi}{2 \sqrt{3}} \frac{z-\frac{7 \pi}{32 \sqrt{3}}}{\left(z-\frac{\pi}{2 \sqrt{3}}\right)^{2}} .
\end{aligned}
$$

The parameter

$$
\Lambda=\frac{2 \pi}{3}(1-r)\left(\frac{L_{x}}{d}\right)^{2}
$$

which appears in the right hand side of Eq. (3), is the hydrodynamic inelastic heat loss parameter. Notice that it can be made arbitrary large (by taking large enough $\left.L_{x} / d\right)$, no matter how small the inelasticity $q=(1-r) / 2$ is.

Now we specify the boundary conditions for Eq. (3). At the elastic walls $x=0$ and $y= \pm \Delta / 2$ the normal component of the heat flux must vanish. In terms of the inverse density $z$ one has $\nabla_{n} z=0$ at these three walls. Here index $n$ denotes the gradient component normal to the wall. Alternatively, for the periodic boundary conditions we should demand $z(x, y+2 \pi / \Delta)=z(x, y)$. The constant temperature at the thermal wall $x=1$ yields the simple condition

$$
z(x=1, y)=\text { const }
$$

with an a priori unknown constant. As the total number of particles $N$ is fixed, the normalization condition

$$
\frac{1}{\Delta} \int_{0}^{1} \int_{-\Delta / 2}^{\Delta / 2} \frac{d x d y}{z(x, y)}=f
$$

should be imposed, where $f=\langle n\rangle / n_{c}$ is the area fraction of the grains and $\langle n\rangle=N /\left(L_{x} L_{y}\right)$ is the average number density of the grains.

Equation (3) with the boundary conditions at the four walls and Eq. (7) make a complete set. Notice that the steady-state density distribution is independent of the wall temperature $T_{0}$ [4]. The wall temperature only sets the scale of the temperature profile in the system, and affects the steady-state pressure. The governing parameters of the system are the scaled numbers $\Lambda, f$ and $\Delta$. For a laterally infinite system, $\Delta \rightarrow \infty$, only two governing parameters $\Lambda$ and $f$ remain in the hydrostatic formulation. 
Let us define the scaled temperature $\tilde{T}=T / T_{0}$ and pressure

$$
\tilde{p}=\frac{p}{n_{c} T_{0}}=\tilde{T} \Pi(z)
$$

where

$$
\Pi(z)=\frac{1+2 G(z)}{z} .
$$

Once the steady state density profile and the (uniform) steady state pressure $\tilde{p}$ are found, Eq. (8) determines the steady state temperature profile $\tilde{T}(x, y)$.

The vector field $F(z) \nabla z$ entering Eq. (3) is, up to a sign, the scaled heat flux. Equation (3) gets simpler if we introduce, as a new variable, the scalar potential of the heat flux: $\psi=\int{ }^{z} F\left(z^{\prime}\right) d z^{\prime}$. To avoid a divergence of the integral at infinity, we account for the diverging part directly, by extracting the first two terms of expansion of $F(z)$ at $z \rightarrow \infty$ :

$$
F(z)=\frac{\sqrt{3}}{2} z^{1 / 2}-\frac{7 \pi}{64} z^{-1 / 2}+f(z) .
$$

The integral of $f(z)$ already converges at infinity, and we obtain

$$
\psi(z)=\frac{1}{\sqrt{3}} z^{3 / 2}+\frac{7 \pi}{32} z^{1 / 2}-\int_{z}^{\infty} f(z) d z,
$$

The potential $\psi$ grows monotonically with $z$, see Fig. [1] Now Eq. (3) becomes a nonlinear Poisson equation [9]:

$$
\nabla^{2} \psi=\Lambda Q(\psi)
$$

where by $Q(\psi)$ we actually mean $Q[z(\psi)]$ here and in the following. The function $Q(\psi)$ is depicted in Fig. 2 We will be dealing with Eq. (12) throughout the paper. The boundary conditions for $\psi(x, y)$ are identical to the boundary conditions for $z(x, y)$ :

$$
\begin{aligned}
\left.\frac{\partial \psi(x, y)}{\partial x}\right|_{x=0} & =0, \\
\psi(1, y) & =\text { const },
\end{aligned}
$$

supplemented by either no-flux, or periodic boundary condition at the walls $y= \pm \Delta / 2$. Note that $z$ is assumed to be expressed through $\psi$ in Eq. (7).

Importantly, $Q(\psi)$ decreases with an increase of $\psi$ at large enough $\psi$. (In the dilute limit, $\psi \gg 1$, one has $Q(\psi) \propto \psi^{-1 / 3}$.) This implies non-uniqueness of steady state solutions of Eq. (12) [15], and opens the way to phase separation and coexistence.

\section{B. Stripe states, spinodal line and critical point}

The simplest steady state of the system is the "stripe": a laterally uniform state which corresponds to the $y$ independent solution $z=Z(x)$ [2], or $\psi=\Psi(x)$. It is described by the equation

$$
\Psi^{\prime \prime}=\Lambda Q(\Psi)
$$

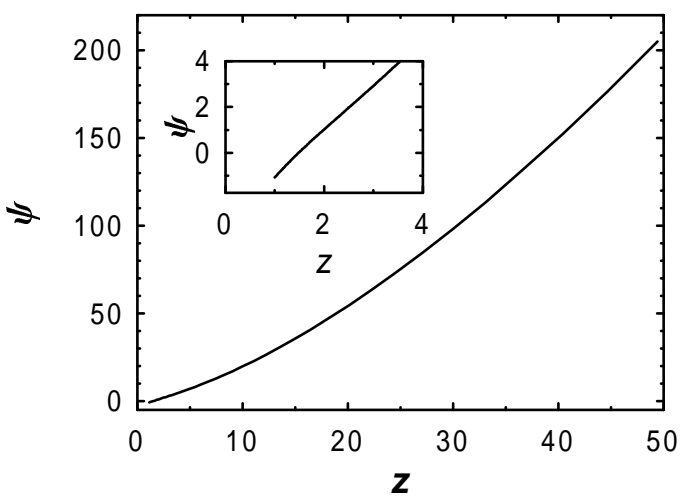

FIG. 1: The effective heat flux potential $\psi$ versus the inverse scaled density $z$. The inset shows a blowup of the region of $1<z<4$.

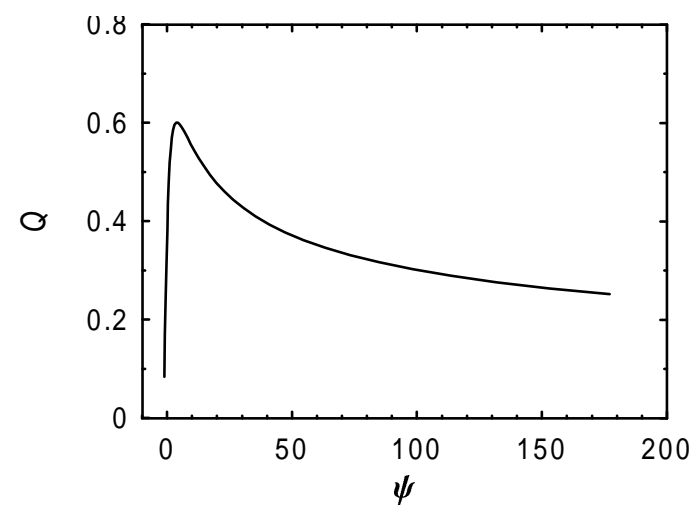

FIG. 2: The effective heat loss function $Q(\psi)$ which appears on the right hand side of Eq. (12).

with the boundary condition

$$
\Psi^{\prime}(0)=0
$$

and normalization condition

$$
\int_{0}^{1} \frac{d x}{Z[\Psi(x)]}=f
$$

Here and in the following the primes denote the $x$ derivatives. As Eq. (15) does not include the first derivative $\Psi^{\prime}(x)$, it has "energy" integral and therefore is integrable. A numerical solution however, is more practical. Figure 3 gives an example of the density profile of the stripe state in terms of the scaled density $n(x) / n_{c}=Z^{-1}(x)$ and the auxiliary functions $\Psi(x)$ and $Z(x)$ for $\Lambda=344.2$ and $f=0.095$, corresponding to the critical point of the phase separation (see below).

The stripe state problem (15)-(17) can be recast into a more convenient initial value problem if we use, instead of the normalization condition (17), a boundary condition

$$
\Psi(0)=a .
$$

Indeed, the initial value problem defined by Eqs. (15), (16) and (18) has a unique solution $\Psi(x, a, \Lambda)$. Having 


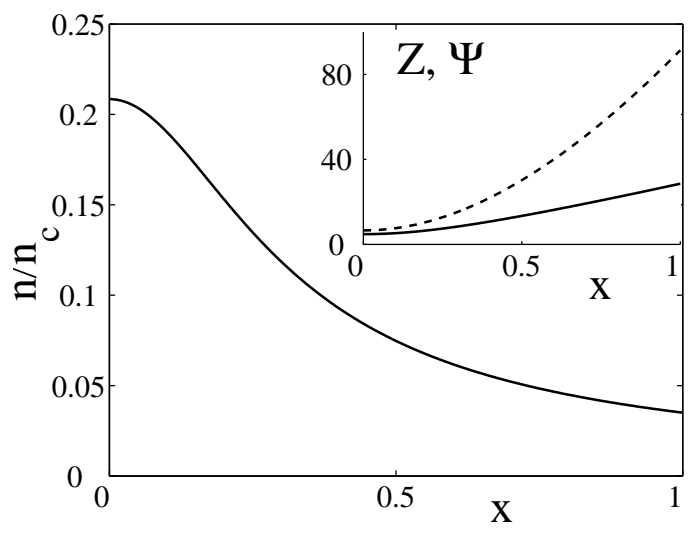

FIG. 3: Spatial profiles of the stripe state for $\Lambda=344.2$ and $f=0.095$, which are the critical values of $\Lambda$ and $f$ for the constitutive relations (4). Shown in the main part of the figure is the scaled density $n(x) / n_{c}=Z^{-1}(x)$. The inset shows $Z(x)$ (the solid line) and the heat flux potential $\Psi(x)$ (the dashed line), obtained by solving Eqs. (15)-(17).

found it, one can calculate the area fraction $f=f(a, \Lambda)$ from Eq. (17). Importantly, for the Enskog-type constitutive relations (4), $f(a, \Lambda)$ turns out to be a monotonic function of $a$ for any fixed $\Lambda$. This enables one to use the pair of numbers $(a, \Lambda)$ instead of $(f, \Lambda)$ for the parametrization of all possible stripe states. The parametrization $(a, \Lambda)$ will be often used in the following.

In a wide region of the parameter space $(f, \Lambda, \Delta)$ the stripe state undergoes a phase separation instability and gives way to a laterally asymmetric state $[4,5,6,6,7,8,9$, 10]. The instability is driven by negative compressibility of the stripe state in the lateral direction [6, 7], resulting from energy loss in particle collisions. Let $P=\tilde{T} \Pi(Z)$ be the scaled steady-state gas pressure of the stripe state. In the limit of $\Delta \rightarrow \infty$, the spinodal region in the $(f, \Lambda)$ plane is defined by the condition $(\partial P / \partial f)_{\Lambda}<0$. As $P$ is constant in space, it can be conveniently computed at the thermal wall $x=1[\underline{6}]$. Here $\tilde{T}=1$ and therefore

$$
P=P(a, \Lambda)=\left.\Pi[z(\psi)]\right|_{\psi=\Psi(1, a, \Lambda)} .
$$

Alternatively,

$$
P(f, \Lambda)=\frac{1+2 G\left[Z_{1}(f, \Lambda)\right]}{Z_{1}(f, \Lambda)},
$$

where $Z_{1}=Z(x=1)$. The spinodal line of the phase separation (again, in the limit of $\Delta \rightarrow \infty$ ) is determined by the condition

$$
\frac{\partial P(f, \Lambda)}{\partial f}=0 .
$$

Solving Eqs. (15)-(18), we computed from Eq. (20) the $P(f)$ curves at different $\Lambda$, see Fig. 4 . These computations yield a critical point $\left(f_{c}, P_{c}\right)$ [equivalently, $\left(f_{c}, \Lambda_{c}\right)$,
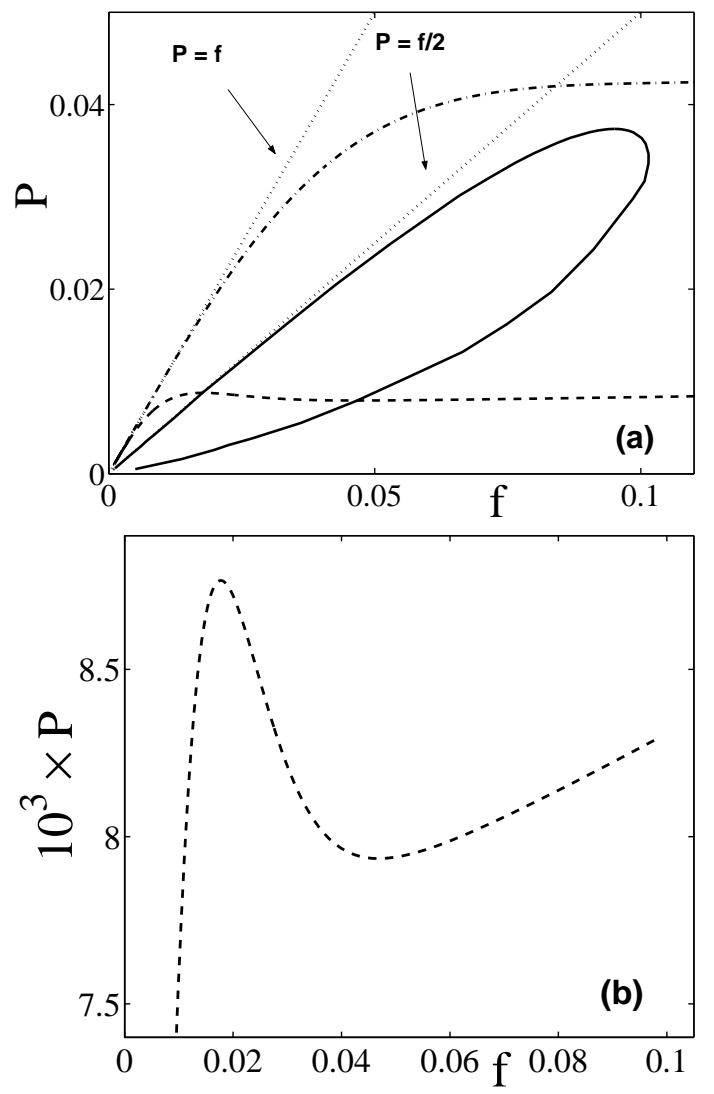

FIG. 4: The spinodal line (the solid line) and the $P(f)$ curves for the stripe state, for $\Lambda=5 \cdot 10^{3}$ and $\Lambda=280$ (the dashed and dash-dotted lines, respectively) (a). The two dotted straight lines are the dilute-limit asymptotes $P_{1}(\Lambda, f)=f$ and $P_{2}(\Lambda, f)=f / 2(\mathrm{a})$. Figure b shows the $P(f)$ curve for $\Lambda=5 \cdot 10^{3}$ in a different scale, to make the region of negative compressibility $d P / d f<0$ more visible.

or $\left.\left(a_{c}, \Lambda_{c}\right)\right]$. For $\Lambda<\Lambda_{c}$ the pressure $P$ increases monotonically with $f$ (like in an elastic gas), so there is no phase separation instability. For $\Lambda>\Lambda_{c}$ the pressure $P$ versus $f$ has a non-monotonic part. The maximum and minimum points of $P(f)$ at different $\Lambda$ yield the spinodal line. This line in the $(f, P)$-plane is shown by the solid curve in Fig. 4h. Figure 4a also shows two straight lines. These are the dilute-limit asymptotes of $P(f)$ and of the low-density branch of the spinodal line, respectively (see the next subsection). Figure $4 \mathrm{~b}$ shows, on a different scale, the $P(f)$-dependence for a fixed $\Lambda$ within the spinodal region, to make the region of negative lateral compressibility more visible. Figure [5]depicts a part of the spinodal line, together with the asymptotics of the spinodal and binodal (coexistence) lines in the vicinity of the critical point, found analytically in Section 3.

Now let us again use the $a$-parametrization of the stripe solution. Equation (21) for the spinodal line is equivalent to

$$
\frac{\partial P(a, \Lambda)}{\partial a}=0
$$



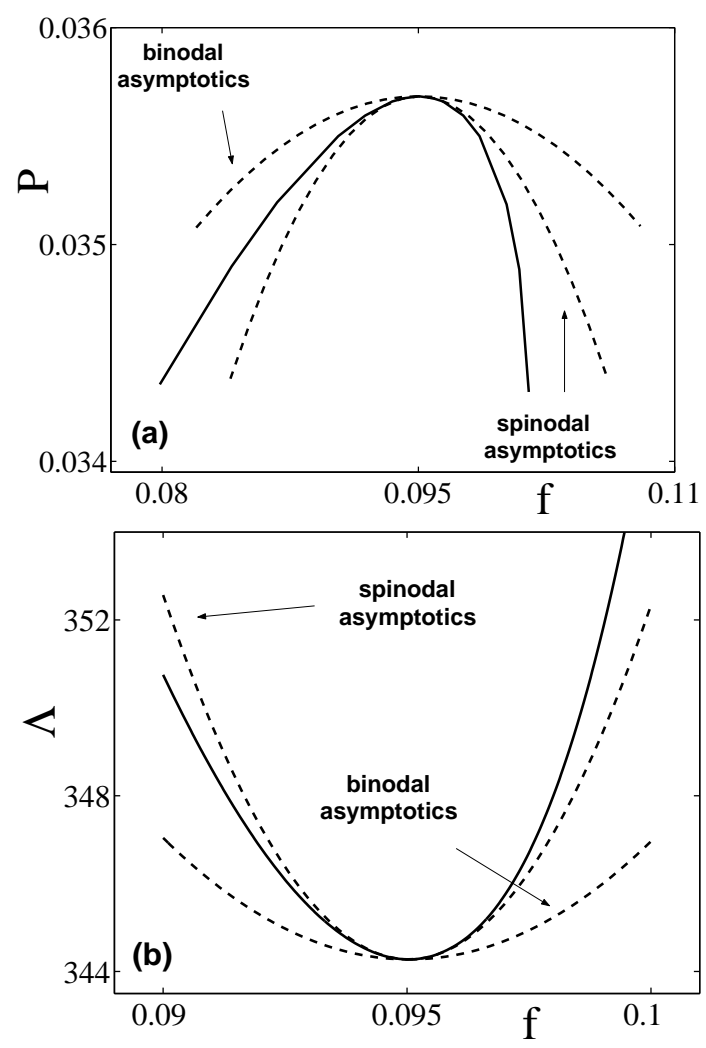

FIG. 5: The spinodal line (the solid line) and the spinodal and binodal asymptotics close to the critical point (the dashed lines) in the variables $f, P(\mathrm{a})$ and $f, \Lambda$ (b).

The critical point is the merging point of the maximum and minimum points of the $P(f)$ [or $P(a)$ ] curve at different $\Lambda$. Therefore, the critical point is defined by the following two conditions:

$$
\begin{gathered}
\frac{\partial P(a, \Lambda)}{\partial a}\left(a_{c}, \Lambda_{c}\right)=0, \\
\frac{\partial^{2} P(a, \Lambda)}{\partial a^{2}}\left(a_{c}, \Lambda_{c}\right)=0 .
\end{gathered}
$$

For an assembly of hard spheres below the freezing point one has $(\partial p / \partial n)_{T}>0$, which follows $\left.(d P / d Z)\right|_{Z=Z_{1}(a, \Lambda)} \neq 0$. Therefore, Eq. (22) for the spinodal line is equivalent to

$$
\frac{\partial \Psi(1, a, \Lambda)}{\partial a}=0
$$

while Eqs. (23) and (24) for the critical point can be rewritten as

$$
\begin{aligned}
\frac{\partial \Psi}{\partial a}\left(1, a_{c}, \Lambda_{c}\right) & =0, \\
\frac{\partial^{2} \Psi}{\partial a^{2}}\left(1, a_{c}, \Lambda_{c}\right) & =0
\end{aligned}
$$

(recall that the first argument of function $\Psi$ and of its derivatives stands for $x$ ). Solving Eqs. (15), (16), (18),
(26) and (27) numerically, we find the critical point

$$
\begin{aligned}
a_{c} & =6.580 \ldots, \\
\Lambda_{c} & =344.2 \ldots
\end{aligned}
$$

Using Eqs. (17) and (20), we find the critical point in the variables $f, P$ :

$$
\begin{aligned}
f_{c} & =f\left(a_{c}, \Lambda_{c}\right)=0.0950 \ldots, \\
P_{c} & =P\left(a_{c}, \Lambda_{c}\right)=0.0373 \ldots
\end{aligned}
$$

We checked that the maximum density of the stripe

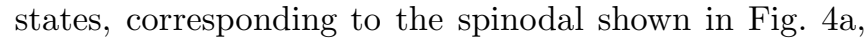
is less than $0.5 n_{c}$, that is within the assumed validity domain of the constitutive relations (4). At the critical point itself the maximum scaled density of the stripe state is $n\left(x=0, a_{c}, \Lambda_{c}\right) / n_{c}=1 / Z\left(x=0, a_{c}, \Lambda_{c}\right)=$ $0.2086 \ldots$, a moderate value.

The critical point, predicted by the Enskog-type granular hydrodynamics, agrees fairly well with molecular dynamic simulations by Soto et al. 7, [10]. The issues of accuracy of the hydrodynamic results are discussed in Section 5 .

\section{Dilute-gas limit}

In the dilute-gas limit, $Z \gg 1$, we can obtain the two

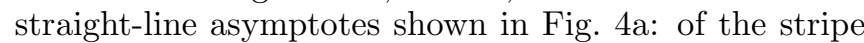
pressure $P(f, \Lambda)$ and of the low-density branch of the spinodal line. Here the stripe state equation is

$$
\frac{d^{2}}{d \bar{x}^{2}} Z^{3 / 2}=3 Z^{-1 / 2}
$$

where a different rescaling of the coordinate is introduced: $\bar{x}=x \sqrt{\Lambda}$. (Notice that in the physical coordinate $x_{\text {phys }}$ the new rescaling does not include $L_{x}$.) The boundary conditions are

$$
Z(\bar{x}=0)=Z_{0} \quad \text { and } \quad \frac{d Z}{d \bar{x}}(\bar{x}=0)=0,
$$

where $Z_{0}$ is related to $f$ and $\Lambda$ by the normalization condition $\int_{0}^{\sqrt{\Lambda}} Z^{-1} d \bar{x}=f \Lambda^{1 / 2}$. The solution to this problem is [a]

$$
\bar{x}=\frac{Z_{0}}{2}\left(\operatorname{arccosh} \sqrt{\zeta}+\sqrt{\zeta^{2}-\zeta}\right),
$$

where $\zeta=Z / Z_{0}$ and

$$
Z_{0}=\frac{4 \Lambda^{1 / 2}}{2 f \Lambda^{1 / 2}+\sinh \left(2 f \Lambda^{1 / 2}\right)} .
$$

In the dimensional units the density profile (33) is determined by a single parameter: $\xi=f \Lambda^{1 / 2}$. The scaled pressure of the stripe state is

$$
P=\frac{1}{Z_{1}}=\frac{2 \xi+\sinh (2 \xi)}{4 \Lambda^{1 / 2} \cosh ^{2} \xi},
$$


where $Z_{1}=Z(x=1)=Z_{0} \cosh ^{2} \xi$. Assuming $\xi \ll$ 1 , we obtain from Eq. (35) the dilute-limit asymptote $P(f, \Lambda)=f$.

The low-density asymptote of the spinodal is determined from the condition

$$
\left(\frac{\partial P}{\partial f}\right)_{\Lambda}=\Lambda^{1 / 2}\left(\frac{\partial P}{\partial \xi}\right)_{\Lambda}=0
$$

This equation, together with Eq. (35), yields $\operatorname{coth}(\xi)=\xi$ [6]. Substituting this result back into Eq. [35), we obtain $P(f, \Lambda)=f / 2$.

Using the dilute-limit Eqs. (33) and (34), one can verify that validity of granular hydrodynamics as an accurate leading order theory in this problem indeed demands nearly elastic collisions (see also Ref. 2] ). Equation (33) implies that the inverse scaled density $Z(x)$ can be presented as $Z=Z_{0} \mathcal{F}\left(\bar{x} / Z_{0}\right)$, where function $\mathcal{F}$ does not include any additional parameters. Therefore, the characteristic scale of inhomogeneity of the stripe state in the $x$-direction in the rescaled coordinate $\bar{x}$ is of the order of $Z_{0}$. Going back to the physical coordinate, $\bar{x}=$ $\Lambda^{1 / 2} x_{\text {phys }} / L_{x} \sim q^{1 / 2} x_{\text {phys }} / d$, we see that the inhomogeneity scale is of the order of $Z_{0} d / q^{1 / 2} \sim\left(n_{0} d q^{1 / 2}\right)^{-1}$. For hydrodynamics to be valid, this quantity should be much greater than the characteristic mean free path of the particles $\bar{l}$ which is of the order of $\left(n_{0} d\right)^{-1}$. Therefore, the validity of hydrodynamics in this system requires $q^{1 / 2} \ll 1$, quite a stringent condition. An additional discussion of this condition is presented in Sec. V.

\section{SPINODAL AND BINODAL LINES IN THE VICINITY OF THE CRITICAL POINT}

\section{A. Mathematical preliminaries}

In the vicinity of the critical point $a_{c}, \Lambda_{c}$ (at $\Lambda>\Lambda_{c}$ ) the two-dimensional, phase separated state is very close to the one-dimensional stripe state. Therefore, the spindal and binodal lines can be obtained by expanding $\psi(x, y)$ around the stripe-state solution $\Psi(x, a, \Lambda)$ in the power series of $a-a_{c}$ and $\Lambda-\Lambda_{c}$. For brevity of notation the subscripts $a$ and $\Lambda$ will denote the partial derivatives $\partial / \partial a$ and $\partial / \partial \Lambda$, respectively, while the prime ' will stand for the partial derivative $\partial / \partial x$ as before. As will become clear shortly, the following functions of $x$ at the critical point need to be computed:

$$
\Psi^{\prime}, \quad \Psi_{a}, \quad \Psi_{\Lambda}, \quad \Psi_{a \Lambda}, \quad \Psi_{a a} \text { and } \Psi_{a a a} .
$$

As the stripe solution $\Psi(x, a, \Lambda)$ is available in quadrature, the derivatives of $\Psi$ with respect to parameters $a$ and $\Lambda$ are known. More practical, however, is a different approach. As shown in Appendix, all these functions [and an additional function $\Phi(x)$ that we will need, see Eq. (60) below] can be expressed as solutions of the linear differential equation

$$
w^{\prime \prime}(x)-\Lambda_{c} Q_{\psi}\left[\Psi\left(x, a_{c}, \Lambda_{c}\right)\right] w(x)=S(x)
$$

with different source terms $S(x)$ and different boundary conditions. For the first derivatives, $\Psi^{\prime}$ and $\Psi_{a}$, the source term vanishes. Therefore, the rest of the functions can be expressed through $\Psi^{\prime}$ and $\Psi_{a}$, see Appendix.

\section{B. Spinodal line in the vicinity of the critical point}

It follows from Eqs. (26) and (27) that the first nonvanishing term in the expansion of $\Psi(x, a, \Lambda)$ in the powers of $a-a_{c}$ at the critical point is the cubic term $\left(a-a_{c}\right)^{3}$. Therefore, we should keep the following terms in the expansion:

$$
\begin{array}{r}
\Psi(x, a, \Lambda)=\Psi\left(x, a_{c}, \Lambda_{c}\right)+\Lambda_{c} \Psi_{\Lambda}\left(x, a_{c}, \Lambda_{c}\right) \delta \\
+a_{c} \Psi_{a}\left(x, a_{c}, \Lambda_{c}\right) u+\frac{a_{c}^{2}}{2} \Psi_{a a}\left(x, a_{c}, \Lambda_{c}\right) u^{2} \\
+a_{c} \Lambda_{c} \Psi_{a \Lambda}\left(x, a_{c}, \Lambda_{c}\right) u \delta+\frac{a_{c}^{3}}{6} \Psi_{a a a}\left(x, a_{c}, \Lambda_{c}\right) u^{3},
\end{array}
$$

where an order parameter $u=a / a_{c}-1$ and control parameter $\delta=\Lambda / \Lambda_{c}-1$ have been introduced.

To obtain the equation of the spinodal line we differentiate Eq. (37) with respect to $a$, put $x=1$ and use Eqs. (25)-(27). The result is

$$
\delta=-\frac{a_{c}^{2} \Psi_{a a a}\left(1, a_{c}, \Lambda_{c}\right)}{2 \Lambda_{c} \Psi_{a \Lambda}\left(1, a_{c}, \Lambda_{c}\right)} u^{2},
$$

or

$$
\Lambda-\Lambda_{c}=A_{1}\left(a-a_{c}\right)^{2},
$$

where

$$
A_{1}=-\frac{\Psi_{a a a}\left(1, a_{c}, \Lambda_{c}\right)}{2 \Psi_{a \Lambda}\left(1, a_{c}, \Lambda_{c}\right)} .
$$

One can see from Eq. (38) that, close to the critical point, $\delta=\mathcal{O}\left(u^{2}\right)$. That is why we could neglect the term proportional to $\Psi_{\Lambda \Lambda} \delta^{2}=\mathcal{O}\left(u^{4}\right)$ in Eq. (37).

The coefficients $\Psi_{a a a}\left(1, a_{c}, \Lambda_{c}\right)$ and $\Psi_{a \Lambda}\left(1, a_{c}, \Lambda_{c}\right)$ can be computed numerically, see Appendix:

$$
\begin{aligned}
\Psi_{\text {aaa }}\left(1, a_{c}, \Lambda_{c}\right) & =0.02434 \ldots, \\
\Psi_{a \Lambda}\left(1, a_{c}, \Lambda_{c}\right) & =-0.001926 \ldots,
\end{aligned}
$$

so $A_{1}=6.317 \ldots$.

Eq. (39) can be rewritten in terms of $\Lambda$ and $f$. Using Eq. (17), we expand $Z(x, a, \Lambda)$ near the critical stripe solution $Z_{c}(x) \equiv Z\left(x, a_{c}, \Lambda_{c}\right)$ up to the first order in $a-a_{c}$ (which suffices close to the critical point) and obtain

$$
f-f_{c}=f_{a}\left(a_{c}, \Lambda_{c}\right)\left(a-a_{c}\right),
$$

where

$$
f_{a}\left(a_{c}, \Lambda_{c}\right)=-\int_{0}^{1} \frac{\Psi_{a}\left(x, a_{c}, \Lambda_{c}\right)}{\left[Z_{c}(x)\right]^{2} F\left(Z_{c}(x)\right)} d x .
$$


Evaluating this integral numerically, we obtain $f_{a}\left(a_{c}, \Lambda_{c}\right)=-0.004396 \ldots$. As the result, Eq. (39) can be rewritten as

$$
\Lambda-\Lambda_{c}=A_{2}\left(f-f_{c}\right)^{2},
$$

where

$$
A_{2}=A_{1}\left[f_{a}\left(a_{c}, \Lambda_{c}\right)\right]^{-2}=3.268 \ldots \cdot 10^{5} .
$$

Now we compute the spinodal line in the $f, P$-plane. Expanding Eq. (19) in the vicinity of $z=Z\left(1, a_{c}, \Lambda_{c}\right)$, we obtain

$$
P=P_{c}+\Pi_{\psi}^{(c)} \Psi_{\Lambda}\left(1, a_{c}, \Lambda_{c}\right)\left(\Lambda-\Lambda_{c}\right)+\ldots,
$$

where the higher-order terms are negligible, and

$$
\Pi_{\psi}^{(c)}=\left.\left(\frac{1}{F(z)} \frac{d \Pi}{d z}\right)\right|_{z=z\left[\Psi\left(1, a_{c}, \Lambda_{c}\right)\right]}=-2.969 \ldots \cdot 10^{-4} .
$$

The negative value of $\Pi_{\psi}^{(c)}$ is a consequence of our definitions of $z$ and $\psi$ and of the condition $(\partial p / \partial n)_{T}>0$. Combining Eqs. (47) and (45) we obtain:

$$
P-P_{c}=-A_{5}\left(f-f_{c}\right)^{2},
$$

where $A_{5}=-A_{2} \Psi_{\Lambda}\left(1, a_{c}, \Lambda_{c}\right) \Pi_{\psi}^{(c)}$. A numerical calculation (see Appendix) gives $\Psi_{\Lambda}\left(1, a_{c}, \Lambda_{c}\right)=0.2203 \ldots$, therefore $A_{5}=21.38 \ldots$. The spinodal asymptotics (49) and (45) are shown, together with the full spinodal line, in Figs. 5 , and 5 , respectively.

\section{Two-phase coexistence and binodal line in the vicinity of the critical point}

\section{Laterally non-uniform states}

For a steady state with a broken lateral symmetry the function $\psi(x, y)$ depends on its two arguments. In a laterally infinite system the asymptotics of $\psi(x, y)$ at $y \rightarrow \pm \infty$ correspond to two different stripe states. Therefore, it is natural to replace the no-flux or periodic boundary conditions in the lateral direction by the condition

$$
\psi(x, y \rightarrow \pm \infty)=\psi_{ \pm}(x),
$$

where $\psi_{-}(x) \neq \psi_{+}(x)$. One way to solve the problem (12)-(14) and (50) is to introduce an unknown function $a(y)$ so that

$$
\begin{gathered}
\psi(0, y)=a(y), \\
a(y \rightarrow \pm \infty)=a_{ \pm}=\mathrm{const}
\end{gathered}
$$

with $a_{-} \neq a_{+}$. What equation should $a(y)$ satisfy close to the critical point? Here we can look for $\psi(x, y)$ in the form of a weakly and slowly modulated stripe state:

$$
\psi(x, y)=\Psi[x, a(y), \Lambda]+\phi(x, y),
$$

where $a(y)=a_{c}[1+u(y)]$ is a slow function of $y, \phi(x, y)$ is a small correction to $\Psi$, and a $y$-dependent order parameter $u(y) \ll 1$ is introduced. We will see shortly that the characteristic length scale of $a(y)$ (the domain wall width) is of the order of $\delta^{-1 / 2} \sim u^{-1}$, while $\phi \sim u^{3}$. Hence, every $y$-derivative introduces smallness of order $u$. Making the Ansatz (52) in Eq. (12) and neglecting terms of a higher order than $u^{3}$, we arrive at the following linear problem for $\phi(x, y)$ :

$$
\begin{gathered}
\partial_{x}^{2} \phi-\Lambda_{c} Q_{\psi}\left[\Psi_{c}(x)\right] \phi=-a_{c} \Psi_{a}\left(x, a_{c}, \Lambda_{c}\right) \frac{d^{2} u}{d y^{2}}, \\
\phi(0, y)=\partial_{x} \phi(0, y)=0 .
\end{gathered}
$$

The first boundary condition in Eq. (54) elmiminates the arbitrariness in the choice of $\phi(x, y)$, while the second one follows from Eqs. (13) and (16). Additional boundary conditions include

$$
\Psi(1, a, \Lambda)+\phi(1, y)=\text { const }
$$

at the thermal wall [see Eq. (14)], and

$$
\phi(x, y \rightarrow \pm \infty)=0
$$

[see Eq. [50)]. Notice, however, that Eqs. (55) and (56) do not enter the problem (53) and (54) for $\phi(x, y)$. Equation (53) can be solved by separation of variables:

$$
\phi(x, y)=a_{c} \Phi(x) \frac{d^{2} u(y)}{d y^{2}} .
$$

The function $\Phi(x)$ is the solution of the following problem:

$$
\begin{gathered}
\Phi^{\prime \prime}-\Lambda_{c} Q_{\psi}\left[\Psi_{c}(x)\right] \Phi=-\Psi_{a}\left(x, a_{c}, \Lambda_{c}\right), \\
\Phi(0)=\Phi^{\prime}(0)=0
\end{gathered}
$$

which again belongs to the class of equations (36). The solution (see Appendix) is

$$
\begin{aligned}
\Phi(x)= & \frac{1}{\Lambda_{c} Q\left(a_{c}\right)}\left[\Psi_{a}\left(x, a_{c}, \Lambda_{c}\right) \int_{0}^{x} \Psi^{\prime} \Psi_{a} d \xi\right. \\
& \left.-\Psi^{\prime}\left(x, a_{c}, \Lambda_{c}\right) \int_{0}^{x} \Psi_{a}^{2} d \xi\right] .
\end{aligned}
$$

where the functions $\Psi^{\prime}$ and $\Psi_{a}$ under the integrals over $\xi$ have arguments $\xi, a_{c}$ and $\Lambda_{c}$. Now we impose the boundary condition (55):

$$
\Psi(1, a, \Lambda)+a_{c} \Phi(1) \frac{d^{2} u}{d y^{2}}=\text { const },
$$


As the second term in the right side of Eq. (55) is of order $u^{3}$, we should keep terms up to $u^{3}$ in the expansion of $\Psi(x, a, \Lambda)$ near the critical point $a_{c}, \Lambda_{c}$. This expansion has the same form as Eq. (37), with the only difference that now $u$ depends on $y$. Evaluating this expansion at $x=1$ and using the definitions of the critical point [Eqs. (26) and [27)], we arrive at the desired equation for $u(y)$ :

$$
u \delta-A_{3} u^{3}+A_{4} \frac{d^{2} u}{d y^{2}}=\alpha=\text { const },
$$

where

$$
\begin{gathered}
A_{3}=-\frac{a_{c}^{2} \Psi_{a a a}\left(1, a_{c}, \Lambda_{c}\right)}{6 \Lambda_{c} \Psi_{a \Lambda}\left(1, a_{c}, \Lambda_{c}\right)}=\frac{a_{c}^{2} A_{1}}{3 \Lambda_{c}}=0.26485 \ldots, \\
A_{4}=\frac{\Phi(1)}{\Lambda_{c} \Psi_{a \Lambda}\left(1, a_{c}, \Lambda_{c}\right)}=0.5212 \ldots,
\end{gathered}
$$

and [see Eq. (60)]

$$
\Phi(1)=-\frac{\Psi^{\prime}\left(1, a_{c}, \Lambda_{c}\right)}{\Lambda_{c} Q\left(a_{c}\right)} \int_{0}^{1} \Psi_{a}^{2} d x=-0.3457 \ldots
$$

\section{Domain wall and the binodal line}

One integration of Eq. (62) yields:

$$
A_{4}\left(\frac{d u}{d y}\right)^{2}=\frac{A_{3}}{2}\left(u^{2}-\frac{\delta}{A_{3}}\right)^{2}+2 \alpha u+\beta,
$$

where $\beta=$ const. To find the constants $\alpha$ and $\beta$, we are using the boundary conditions in the lateral direction. In an infinite system these are

$$
u(y \rightarrow \pm \infty)=u_{ \pm}=\text {const }
$$

with $u_{-} \neq u_{+}$. Equations (66) and (67) appear in numerous problems of domain wall structure, see for example Ref. [16]. The boundary conditions (67) yield $\alpha=\beta=0$, so the domain wall solution is

$$
u= \pm \sqrt{\frac{\delta}{A_{3}}} \tanh \left[\sqrt{\frac{\delta}{2 A_{4}}}\left(y-y_{0}\right)\right],
$$

where \pm refers to two possible orientations, and the arbitrary constant $y_{0}$ describes the position of the domain wall. The domain wall solution exists only if $\delta>0$, that is $\Lambda>\Lambda_{c}$. Eq. (68) confirms our assumption that the characteristic width of the domain wall is $\mathcal{O}\left(\delta^{-1 / 2}\right)$. Returning for a moment to the physical (unscaled) variables, we see that the domain wall width is $\mathcal{O}\left(L_{x} / \delta^{1 / 2}\right)$ which is much larger than $L_{x}$.

The values of the order parameter far from the domain wall are

$$
u_{ \pm}=\frac{a_{ \pm}}{a_{c}}-1= \pm \sqrt{\frac{\delta}{A_{3}}} .
$$

Equation (69), rewritten in terms of $a$ and $\Lambda$, defines the binodal (coexistence) line:

$$
\Lambda-\Lambda_{c}=\frac{A_{1}}{3}\left(a-a_{c}\right)^{2},
$$

or, in terms of $f$ and $\Lambda$,

$$
\Lambda-\Lambda_{c}=\frac{A_{2}}{3}\left(f-f_{c}\right)^{2} .
$$

Compare these expressions with Eqs. (39) and (45) for the spinodal.

The binodal line can be also expressed in terms of $f$ and $P$. The derivation almost coincides with that for the spinodal line, see the end of Sec. IIIB The only difference is that now we substitute into Eq. (47) the binodal relation (71) rather than the spinodal relation 45). The result is

$$
P-P_{c}=-\frac{A_{5}}{3}\left(f-f_{c}\right)^{2} .
$$

The physical meaning of the binodal asymptotics (172) and (71) is straightforward. Firstly, the two coexisting stripes with $f=f_{1}$ and $f=f_{2}$ have equal pressures: $P\left(f_{1}\right)=P\left(f_{2}\right)$. Secondly, close to the critical point, $f_{1}$ and $f_{2}$ are symmetric with respect to $f_{c}$, that is $\left(f_{1}+\right.$ $\left.f_{2}\right) / 2=f_{c}$. The binodal asymptotics (72) and (71) are depicted in Figs. 57 and 5b, respectively.

Note that our results for the spinodal and binodal lines [see Eqs. (45) and (71)] are consistent with the results obtained by Soto et al. 7, 10] in the framework of their phenomenological "van der Waals equation". Indeed, our Eqs. (45) and (71) have the same quadratic forms as those obtainable from the van der Waals equation [7, 10]. Furthermore, the ratio of the coefficients of the spinodal and binodal lines (45) and (71) equals three, in agreement with what the van der Waals equation predicts. (Note that the coefficients themselves of the van der Waals equations have not been derived yet.) In addition, Soto et al. 10] reported a binodal line found in molecular dynamics simulations of this system for a moderate value of the parameter $\varepsilon=d / L_{x}=0.01$. See Sec. 5 for a discussion of the role of this parameter.

Soto et al. 10 also suggested an interpretation of the binodal line in terms of the "Maxwell's construction". By analogy with the classical van der Waals gas [17], Maxwell's construction can be written as

$$
\int_{f_{1}}^{f_{2}} \frac{P(f, \Lambda)-P\left(f_{1}, \Lambda\right)}{f^{2}} d f=0,
$$

where the factor $f^{2}$ in the denominator comes from equality $d V=d(1 / f)=-d f / f^{2}$, where $V=1 / f$ is the (scaled) specific volume. How does Eq. (73) compare to our result (72)? Consider expression (19) for $P(a, \Lambda)$. Close to the critical point, it can be expanded in the powers of $u$ and $\delta$ :

$$
P=P_{c}+\Lambda_{c} P_{\Lambda} \delta+a_{c} \Lambda_{c} P_{a \Lambda} u \delta+\frac{1}{6} a_{c}^{3} P_{a a a} u^{3}+\ldots,
$$


where all the derivatives of $P$ are evaluated at the critical point. Going over from $u$ to $f-f_{c}$, one obtains

$$
\begin{array}{r}
P=P_{c}+\Lambda_{c} P_{\Lambda} \delta+\frac{\Lambda_{c} P_{a \Lambda} \delta}{f_{a}}\left(f-f_{c}\right) \\
+\frac{P_{a a a}}{6 f_{a}^{3}}\left(f-f_{c}\right)^{3}+\ldots,
\end{array}
$$

where the numerical coefficient $f_{a}$ is given by Eq. (44), and the dots stand for terms of the order of $\left(f-f_{c}\right)^{4}$ and higher. At $\delta>0$ (when phase separation occurs), the coefficient in front of $f-f_{c}$ is negative, while that in front of $\left(f-f_{c}\right)^{3}$ is positive. Note also that, in the vicinity of the critical point, $\delta=\mathcal{O}\left(f-f_{c}\right)^{2}$. Now we substitute Eq. (75), with the higher-order terms neglected, into Eq. (73). In this order of perturbation theory one should put $f=f_{c}$ in the denominator of the integrand. As the result, Eq. (73) reduces to the simple relation $\left(f_{1}+\right.$ $\left.f_{2}\right) / 2=f_{c}$ obtained above. Of course, the reason for this agreement is the closeness to the critical point. In this sense, the Maxwell's construction (73) does not give anything new. Moreover, many different constructions, for example,

$$
\int_{f_{1}}^{f_{2}}\left[P(f, \Lambda)-P\left(f_{1}, \Lambda\right)\right] d f=0,
$$

are equally applicable in the vicinity of the critical point. Of course, the Maxwell's construction would be valuable if it were shown to be true far from the critical point. At present there is no reason to believe this is the case, and the theoretical form of the binodal line far from the critical point remains unknown.

\section{FINITE SIZE EFFECTS: MARGINAL STABILITY AND BIFURCATIONS}

\section{A. Marginal stability surface}

The results presented in Sections 2 and 3 are valid in the limit of $\Delta \rightarrow \infty$. Already in the first paper [4] on the phase separation instability it was found that the instability is suppressed when the aspect ratio of the box $\Delta=L_{y} / L_{x}$ is less than a threshold value $\Delta_{*}(f, \Lambda)$. The physical mechanism of suppression is heat conduction in the lateral direction which tends to erase the lateral temperature (and, therefore, density) inhomogeneity. How to generalize the spinodal line, obtained for $\Delta \rightarrow \infty$, to finite $\Delta$-s? Let us consider a small sinusoidal density perturbation, in the lateral direction, around a stripe state. The fastest growing (or the slowest decaying) perturbation is the one with the longest wavelength, compatible with the boundary conditions in the lateral direction [4, 5, 6]. Consider a three-dimensional parameter space $(f, \Lambda, \Delta)$ and define in it a two-dimensional marginal stability surface $\mathcal{F}(f, \Lambda, \Delta)=0$. By definition, at any point on this surface the growth rate of the longest perturbation is equal to zero. The marginal stability surface represents a natural generalization of the spinodal line. Importantly, this definition reduces to that of the spinodal line as $\Delta \rightarrow \infty$ [see Ref. [6] and Eq. [87) below]. The marginal stability surface can be computed by linearizing Eq. (12) around the stripe state and solving the resulting linear eigenvalue problem. Calculations of this kind were done previously for large $\Lambda$ far from the critical point [4, 5, 6]. Three typical cross-sections of the marginal stability surface are shown in Fig. [6] As expected, the instability region shrinks as $\Delta$ goes down. As the result, the critical point moves toward smaller $f$-s and larger $\Lambda$-s as $\Delta$ decreases. The monotonic dependence of the critical point position on $\Delta$ is explained by the monotonic increase of the lateral heat conduction as $\Delta$ decreases.

The threshold value of the aspect ratio $\Delta_{*}(\Lambda, f)$ has a minimum at some $f[4,6]$. The respective minimum value $\Delta_{\min }$ depends only on $\Lambda$. In this work we performed a systematic investigation of this dependence. We found that $\Delta_{\min }$ goes down monotonically as the parameter $\Lambda-\Lambda_{c}$ is positive and increases. Though this monotonic decrease looks like a power law in the log-log plot, see Fig. [7, it is actually not. Figure $7 \mathrm{~b}$ shows the same dependence on a different scale. Two different asymptotes are clearly seen. The first of them, at $\Lambda \gg 1$, was obtained previously: $\Delta_{\min }(\Lambda)=A \Lambda^{-1 / 2}$, where $A=52.14 \ldots$ [4, 6]. In this regime the eigenfunction of the marginal stability problem is exponentially localized at the elastic wall $x=0$. The second asymptote is valid close to the critical point $\Lambda=\Lambda_{c}$, where $\Delta_{\min }$ diverges: $\Delta_{\min }=42.085 \ldots\left(\Lambda-\Lambda_{c}\right)^{-1 / 2}$. This asymptote is derived analytically in the next subsection.

An additional interesting issue is the change of bifurcation character, predicted by a weakly nonlinear analysis of the steady state problem close to the marginal stability surface. At fixed $\Lambda$, the bifurcation is supercritical on an interval $f_{-}(\Lambda)<f<f_{+}(\Lambda)$ which lies within the spinodal interval $\left(f_{1}, f_{2}\right)$. On the intervals $f_{1}<f<f_{-}$ and $f_{+}<f<f_{2}$ the bifurcation is subcritical [8, [9]. The next subsection addresses the finite size effects in the vicinity of the critical point, where everything can be calculated analytically.

\section{B. Steady states and bifurcation types close to the critical point}

This subsection addresses two-dimensional steady states in a laterally finite system close to the critical point. As $\Delta_{\min }$ diverges at the critical point [see Eq. (90) below], we assume that $\Delta$, though finite, is very large. The starting point here is the same Eq. (62), but on a finite interval $-\Delta / 2 \leq y \leq \Delta / 2$, so we replace the 


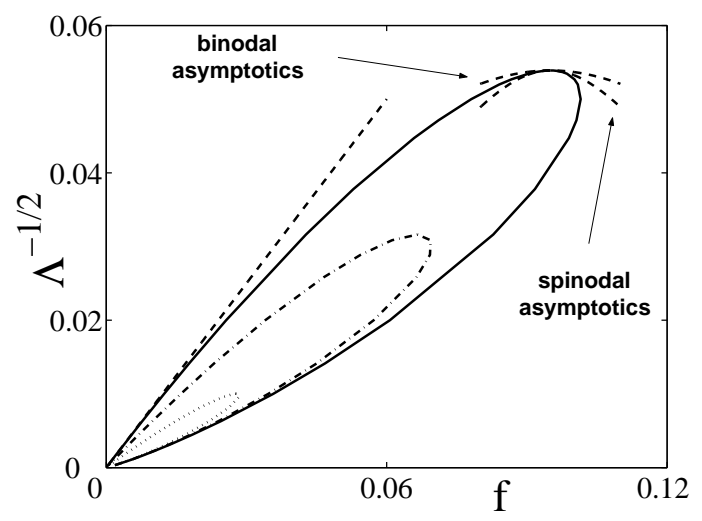

FIG. 6: Cross-sections of the marginal stability surface by three planes: $\Delta=\infty$ (the solid line), $\Delta=1.788$ (the dashdotted line) and $\Delta=0.523$ (the dotted line). The dashed lines show the dilute-limit asymptote $f \Lambda^{1 / 2}=1.1997 \ldots$, obtained analytically [6], and the spinodal and binodal asymptotics near the critical point.

boundary conditions [67) by the no-flux conditions

$$
\left.\frac{d u}{d y}\right|_{y=-\frac{\Delta}{2}}=\left.\frac{d u}{d y}\right|_{y=\frac{\Delta}{2}}=0 .
$$

Periodic boundary conditions can be treated in a similar manner. Integrating Eq. (62) over $y$ from $-\Delta / 2$ to $\Delta / 2$ and using the boundary conditions (77), we determine $\alpha$ and rewrite Eq. (62) as

$$
A_{4} \frac{d^{2} u}{d y^{2}}+u \delta-A_{3} u^{3}-\left\langle u \delta-A_{3} u^{3}\right\rangle=0
$$

where $\langle\ldots\rangle$ denotes spatial averaging:

$$
\langle\ldots\rangle=\frac{1}{\Delta} \int_{-\Delta / 2}^{\Delta / 2}(\ldots) d y .
$$

Introduce the rescaled coordinate $y_{*}=y / \Delta$, order parameter $u_{*}=u \Delta$ and control parameter $\delta_{*}=\Delta^{2} \delta$. Equation (78) keeps its form in the rescaled variables,

$$
A_{4} \frac{d^{2} u_{*}}{d y^{2}}+u_{*} \delta_{*}-A_{3} u_{*}^{3}-\left\langle u_{*}\right\rangle \delta_{*}+A_{3}\left\langle u_{*}^{3}\right\rangle=0
$$

while the boundary conditions become

$$
\frac{d u_{*}}{d y}\left(y_{*}=-1 / 2\right)=\frac{d u_{*}}{d y}\left(y_{*}=1 / 2\right)=0 .
$$

As the aspect ratio $\Delta$ drops out in these variables, a universal description can be obtained.

Obviously, any $y$-independent state $u_{*}=$ const solves the problem (79)-80) (a $y$-independent state is nothing but a stripe state). What is the condition for the appearance of (weakly) $y$-dependent solutions? When a $y$-dependent solution does appear, what is the type of
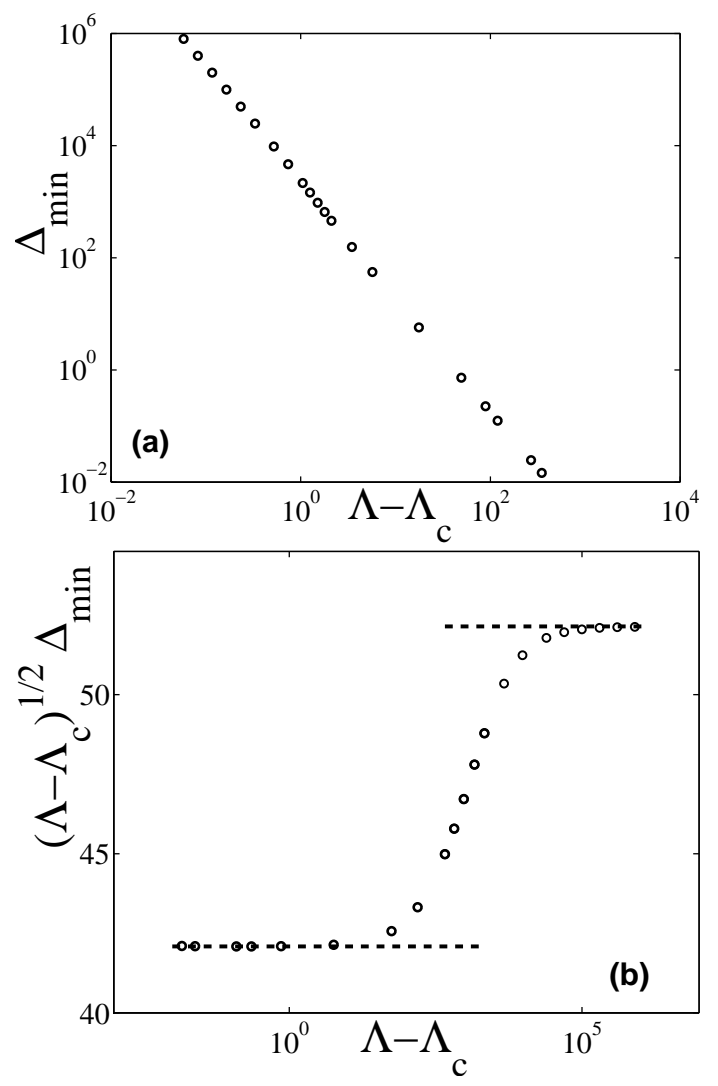

FIG. 7: The dependence of the threshold value of the aspect ratio $\Delta$ for the suppression of the phase separation on the parameter $\Lambda-\Lambda_{c}$ (a). The phase separation instability occurs when $\Delta>\Delta_{\min }(\Lambda)$. Figure b shows that the apparent "straight line" in figure a actually includes two different asymptotes and a crossover between them. The two asymptotes are shown by the dashed lines: the $\Lambda \gg 1$ asymptote, $\Delta_{\min }(\Lambda)=52.14 \ldots \Lambda^{-1 / 2}$, and the asymptote near the critical point, $\Delta_{\min }=\pi\left(\Lambda_{c} A_{4}\right)^{1 / 2}\left(\Lambda-\Lambda_{c}\right)^{-1 / 2}$, where $A_{4}=0.5213 \ldots$. The results of numerical marginal stability analysis are denoted by circles.

bifurcation? To address these questions, we seek for a weakly $y$-dependent solution in the form

$$
u_{*}=\left\langle u_{*}\right\rangle+a_{1} \sin \pi y_{*}+a_{2} \cos 2 \pi y_{*}+\ldots,
$$

We substitute Eq. (81) into Eq. (79) and treat the terms originating from $a_{2} \cos 2 \pi y_{*}$ as small corrections. Expanding up to $\mathcal{O}\left(a_{1}^{3}\right)$, we obtain the following two algebraic equations:

$$
\begin{aligned}
& \delta_{*}-\pi^{2} A_{4}-3 A_{3}\left\langle u_{*}\right\rangle^{2}-\frac{3}{4} A_{3} a_{1}^{2}+3 A_{3}\left\langle u_{*}\right\rangle a_{2}=0, \\
& \frac{3}{2} A_{3}\left\langle u_{*}\right\rangle a_{1}^{2}+\left(\delta_{*}-4 \pi^{2} A_{4}-3 A_{3}\left\langle u_{*}\right\rangle\right) a_{2}=0 .
\end{aligned}
$$

Putting here $a_{1}=a_{2}=0$, we obtain the marginal stability condition

$$
\delta_{*}=3 A_{3} u_{*}^{2}+\pi^{2} A_{4},
$$


where we have omitted the spatial averaging of $u_{*}$, as it becomes trivial on the marginal stability curve. The marginal stability curve is shown, as the thick solid line, in Fig. 8. Now we consider nonzero amplitudes $a_{1}$ and $a_{2}$ in Eq. (82) and eliminate $a_{2}$ in favor of $a_{1}$. Above the marginal stability curve (83), but close to it, we obtain the following equation for the bifurcation curve:

$$
\frac{\delta_{*}-3 A_{3}\left\langle u_{*}\right\rangle^{2}-\pi^{2} A_{4}}{3 A_{3}}=\frac{a_{1}^{2}}{4}\left(1-\frac{2 A_{3}\left\langle u_{*}\right\rangle^{2}}{\pi^{2} A_{4}}\right) .
$$

One can see that, on the marginal stability curve, the bifurcation is either supercritical (when the term in the parentheses in the right hand side of this equation is positive), or subcritical (when this term is negative). The change of character of the bifurcation occurs at the points

$$
\begin{aligned}
\left\langle u_{*}\right\rangle & = \pm\left(\frac{\pi^{2} A_{4}}{2 A_{3}}\right)^{1 / 2}= \pm 3.116 \ldots, \\
\delta_{*} & =\frac{5}{2} \pi^{2} A_{4} \simeq 12.86 \ldots
\end{aligned}
$$

which lie on the marginal stability curve (83), see Fig. 8

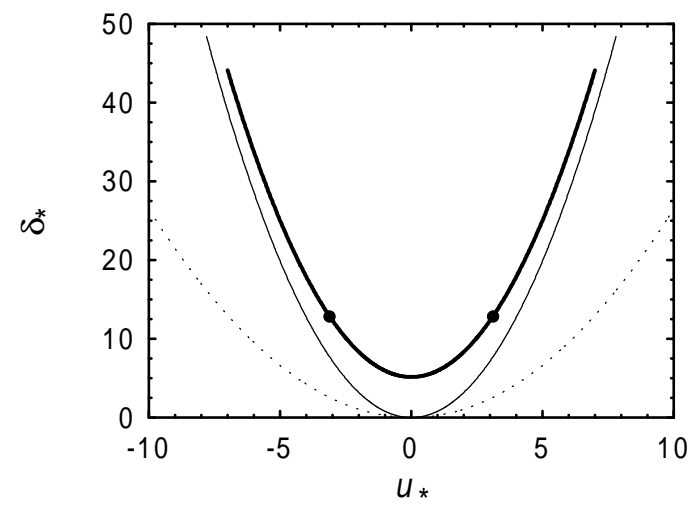

FIG. 8: The marginal stability curve for a laterally finite system (the thick solid line) is shown together with the spinodal and binodal lines for an infinite system (the thin solid line and the thin dotted line, respectively). This is made possible by using the rescaled order parameter $u_{*}$ and control parameter $\delta_{*}$. The two black circles show the points (85) and (86), where the bifurcation changes its character from the supercritical (between the circles) to the subcritical (outside).

Note that the spinodal and binodal lines for an infinite system can be expressed through $u_{*}$ and $\delta_{*}: \delta_{*}=3 A_{3} u_{*}^{2}$ and $\delta_{*}=A_{3} u_{*}^{2}$, respectively. This makes it possible to show all the curves (the spinodal and binodal for an infinite system and the marginal stability curve for a finite system) on the same plot, see Fig. 8

Going back from the rescaled variables $\delta_{*}$ and $u_{*}$ to $\Lambda$ and $a$, we obtain for the marginal stability curve:

$$
\Lambda-\Lambda_{c}=A_{1}\left(a-a_{c}\right)^{2}+\frac{\pi^{2} A_{4} \Lambda_{c}}{\Delta^{2}},
$$

or, in the variables $f, \Lambda$ :

$$
\Lambda-\Lambda_{c}=A_{2}\left(f-f_{c}\right)^{2}+\frac{\pi^{2} A_{4} \Lambda_{c}}{\Delta^{2}} .
$$

The physical meaning of this result becomes transparent when one compares it with Eq. (45) for the spinodal in the infinite system.

Solving Eq. (88) for $\Delta$, we can find the threshold value of the aspect ratio $\Delta_{*}$ at given $f$ and $\Lambda$ :

$$
\Delta_{*}=\frac{\pi A_{4}^{1 / 2} \Lambda_{c}^{1 / 2}}{\left[\Lambda-\Lambda_{c}-A_{2}\left(f-f_{c}\right)^{2}\right]^{1 / 2}} .
$$

The phase separation instability occurs at $\Delta>\Delta_{*}$. One can see that $\Delta_{*}$ diverges on the spinodal line. The minimum value of $\Delta_{*}$ at a given $\Lambda$ corresponds to $f=f_{c}$ :

$$
\Delta_{m i n}=\frac{\pi A_{4}^{1 / 2} \Lambda_{c}^{1 / 2}}{\left(\Lambda-\Lambda_{c}\right)^{1 / 2}} .
$$

This quantity diverges at the critical point, as the lower asymptote in Fig. 7 shows.

Finally, the two points of change of the bifurcation character, [Eqs. (85) and (86)], form a line,

$$
\Lambda-\Lambda_{c}=\frac{5}{3} A_{1}\left(a-a_{c}\right)^{2}
$$

in the plane $a, \Lambda$, which corresponds to the line

$$
\Lambda-\Lambda_{c}=\frac{5}{3} A_{2}\left(f-f_{c}\right)^{2},
$$

in the plane $f, \Lambda$. This line lies within the spinodal interval of the problem corresponding to $\Delta \rightarrow \infty$.

\section{SUMMARY AND DISCUSSION}

In this work we have employed granular hydrostatics to determine the phase diagram of a prototypical driven granular gas which exhibits spontaneous symmetry breaking and van der Waals-like phase separation. We determined the spinodal line and the critical point of the phase separation. We computed the spinodal and binodal (coexistence) lines close to the critical point. Effects of finite lateral size of the confining box have been also addressed. These include determining the line of change of the bifurcation character from supercritical to subcritical.

The shape of the binodal (coexistence) line far from the critical point is still unknown. To handle this problem within the framework of the hydrostatic theory one needs to solve the nonlinear Poisson equation (12) in a laterally infinite box. Most likely, this can only be done numerically, in a sufficiently long box. It is clear, however, that the binodal line in the variables $(a, \Lambda)$ is solely determined by the function $Q(\psi)$ which encapsulates all the necessary information about the equation of state, heat conductivity and inelastic energy loss.

It is worth mentioning that our qualitative results (the phase separation instability, the existence of the critical point and spinodal and binodal lines, the suppression of the instability by the lateral heat conduction and 
the change of bifurcation character) are robust and independent of the small details of the constitutive relations. Furthermore, most of our analytical results are presented in quite a general form which only assumes a Navier-Stokes structure of the hydrodynamic equations (in the limit of nearly elastic collisions). We used the Enskog-type constitutive relations [11] only for computing numerical factors (and for the computations far from the critical point). Obviously, the accuracy of our quantitative results cannot be better then the accuracy of the Enskog-type constitutive relations. Therefore, a 10-15 percent error margin should not be surprising.

There is an important additional aspect that can become crucial when comparing the hydrodynamic theory with molecular dynamic simulations. As was already mentioned, for the hydrodynamics to be quantitatively accurate, the mean free path of the particles must be much less than any hydrodynamic length scale. It is well known that, for a system with a thermal wall, the leading correction to hydrodynamics enters the boundary condition in the form of a temperature jump, proportional to the ratio of the mean free path and the characteristic hydrodynamic length scale [18, 19]. Indeed, within the Knudsen layer, whose size is comparable to the mean free path, hydrodynamics is inapplicable, while the particle velocity distribution significantly deviates from Maxwell distribution: the temperature of the incoming particles is less than that of the outgoing particles [as the outgoing particles have their normal velocity randomized according to a Maxwell distribution with a (fixed) higher temperature]. The effective temperature at the wall, for the purpose of a hydrodynamic description in the bulk (that is, outside the Knudsen layer), is always less than $T_{0}[18,19$, as is indeed observed in MD simulation of this system [2]. As the result, the pressure of the system is reduced (notice that the density does not change in this order; it can change only in the next, Burnett order). The pressure reduction will obviously cause a shift of the critical point. For our numerical results to be sufficiently accurate the parameter $\varepsilon=d / L_{x}$ must be very small, so that the mean free path is indeed much less than the system dimensions. As $\Lambda \sim q \varepsilon^{-2}=$ const (for example, at the critical point $\Lambda=\Lambda_{c}$ ), a very small $\varepsilon$ implies an extremely small inelasticity $q$. Despite the severe limitation intrinsic to it, the nearly elastic case is conceptually important, just because hydrodynamics is supposed to give here a quantitatively accurate leading order theory.

An interesting direction for future work is the phase separation dynamics. Here the hydrostatic equations should give way to the full set of hydrodynamic equations. Close to the critical point, however, a reduced description of the dynamics should be possible. It has been suggested that such a description is provided by the "van der Waals equation" 7, 10]. Though the van der Waals equation does capture qualitatively much of the phenomenology of the phase separation as seen in the MD simulations 7, 10], its systematic derivation from the equations of granular hydrodynamics is still lacking, and the coefficients of the normal form are yet unknown. Importantly, our hydrostatic results close to the critical point, encapsulated in Eq. (62), fully agree with those predicted from the van der Waals normal equation. Furthermore, Eq. (62) provides quantitative relations between the yet unknown coefficients of the van der Waals equation.

Another avenue of future work requires going beyond hydrodynamic description, as it includes two types of fluctuations in this system. The first of them was observed in molecular dynamic simulations inside the spinodal region at $\Lambda=11050$ (that is, far from the critical point) in a wide region of aspect ratios around the finitesize threshold value $\Delta_{*}[9]$. It was found that these fluctuations dominate the dynamics of the system, so they were called giant fluctuations. The second type of fluctuations is expected to occur, by analogy with the classical van der Waals phase transition, in a close vicinity of the critical point. The fluctuations in these two regimes should be describable in the framework of "fluctuating hydrodynamics" of Landau and Lifshitz [19], generalized to granular gases in the limit of nearly elastic collisions. Fluctuating hydrodynamics is a Langevin-type theory which takes into account the discrete character of particles by adding delta-correlated noise terms in the momentum and energy equations [19]. The fluctuations appear in this approach as a hydrodynamic response of the system to the Langevin noise. Unfortunately, this exciting direction of work is hindered by the fact that the Langevin term, which accounts for the discreteness of the inelastic energy loss in the energy equation, has yet to be calculated [9].

Finally, it was assumed throughout the paper that the granulate is driven by a thermal wall. In experiment a rapidly vibrating wall is usually used. Though qualitatively similar, the phase diagram of the case of a rapidly vibrating wall can be quantitatively different [4, 5, 8].

\section{Acknowledgments}

This research was partially supported by the Israel Science Foundation (Grant No. 180/02), by the Russian Foundation for Basic Research (Grant No. 02-01-00734), and by the Forchheimer Foundation.

\section{APPENDIX. COMPUTING THE COEFFICIENTS}

Consider the stripe solution $\Psi(x, a, \Lambda)$. The expansions in the vicinity of the critical point, used throughout the paper, include several derivatives of this function which need to be evaluated. These are $\Psi^{\prime}, \Psi_{a}, \Psi_{a a}$, $\Psi_{\Lambda}, \Psi_{\Lambda a}, \Psi_{a a a}$, and additional function $\Phi$ [see Eqs. (58) and [59] ], all of them evaluated at the critical point $a=a_{c}, \Lambda=\Lambda_{c}$. One can easily show that each of these functions is a solution of the linear problem

$$
w^{\prime \prime}(x)-\Lambda_{c} Q_{\psi}\left(\Psi_{c}\right) w(x)=S(x)
$$




\begin{tabular}{|c|c|c|c|}
\hline \multirow[t]{2}{*}{$w(x)$} & \multirow[t]{2}{*}{$S(x)$} & \multicolumn{2}{|c|}{ Initial conditions at $x=0$} \\
\hline & & $w(0)$ & $w^{\prime}(0)$ \\
\hline$\Psi^{\prime}\left(x, a_{c}, \Lambda_{c}\right)$ & 0 & 0 & $\Lambda_{c} Q\left(a_{c}\right)$ \\
\hline$\Psi_{a}\left(x, a_{c}, \Lambda_{c}\right)$ & 0 & 1 & 0 \\
\hline$\Psi_{\Lambda}\left(x, a_{c}, \Lambda_{c}\right)$ & $Q\left(\Psi_{c}\right)$ & 0 & 0 \\
\hline$\Psi_{a a}\left(x, a_{c}, \Lambda_{c}\right)$ & $\Lambda_{c} Q_{\psi \psi}\left(\Psi_{c}\right) \Psi_{a}^{2}$ & 0 & 0 \\
\hline$\Psi_{\Lambda a}\left(x, a_{c}, \Lambda_{c}\right)$ & $\Lambda_{c} Q_{\psi \psi}\left(\Psi_{c}\right) \Psi_{\Lambda} \Psi_{a}+Q_{\psi}\left(\Psi_{c}\right) \Psi_{a}$ & 0 & 0 \\
\hline$\Psi_{a a a}\left(x, a_{c}, \Lambda_{c}\right)$ & $3 \Lambda_{c} Q_{\psi \psi}\left(\Psi_{c}\right) \Psi_{a} \Psi_{a a}+\Lambda_{c} Q_{\psi \psi \psi}\left(\Psi_{c}\right) \Psi_{a}^{3}$ & 0 & 0 \\
\hline$\Phi\left(x, a_{c}, \Lambda_{c}\right)$ & $-\Psi_{a}$ & 0 & 0 \\
\hline
\end{tabular}

TABLE I: The source terms $S(x)$ and the initial conditions at $x=0$ for Eq. (93), for each of the auxiliary functions shown in the first column. The functions in the first and second columns have the same arguments.

with different source terms $S(x)$ and different initial conditions at $x=0$. The source terms and initial conditions are listed in Table. [

Let us show, as an example, the derivation of $S(x)$ and of the initial conditions for two of the functions: $\Psi_{a}$ and $\Psi_{\Lambda a}$. The starting point is Eq. (15) with the initial conditions (16) and (18) for the stripe solution $\Psi(x, a, \Lambda)$. The stripe solution depends on $a$ and $\Lambda$ because they enter either the equation, or the initial conditions. Let us differentiate the both sides of Eq. (15) with respect to a. We obtain

$$
\Psi_{a}^{\prime \prime}(x, a, \Lambda)-\left.\Lambda \frac{d Q}{d \psi}\right|_{\psi=\Psi(x, a, \Lambda)} \Psi_{a}(x, a, \Lambda)=0 .
$$

Once $\Psi(x, a, \Lambda)$ is known, Eq. (94) is a linear differential equation for $\Psi_{a}$. Now let us differentiate, with respect to $a$, Eqs. (16) and (18). We obtain the relations

$$
\Psi_{a}(0, a, \Lambda)=1 \text { and } \Psi_{a}^{\prime}(0, a, \Lambda)=0,
$$

which serve as the initial conditions for the same function $\Psi_{a}$. As we see, the source term $S(x)$ vanishes in this case.

Differentiating Eqs. (94) and (95) with respect to $\Lambda$, we arrive at the following problem for the function $\Psi_{a \Lambda}$ :

$$
\begin{gathered}
\Psi_{a \Lambda}^{\prime \prime}(x, a, \Lambda)-\left.\Lambda \frac{d Q}{d \psi}\right|_{\psi=\Psi(x, a, \Lambda)} \Psi_{a \Lambda}(x, a, \Lambda)= \\
\left.\frac{d Q}{d \psi}\right|_{\psi=\Psi(x, a, \Lambda)} \Psi_{a}(x, a, \Lambda)+ \\
\left.\Lambda \frac{d^{2} Q}{d \psi^{2}}\right|_{\psi=\Psi(x, a, \Lambda)} \Psi_{\Lambda}(x, a, \Lambda) \Psi_{a}(x, a, \Lambda), \\
\Psi_{a \Lambda}(0, a, \Lambda)=0,
\end{gathered}
$$

$$
\Psi_{a \Lambda}^{\prime}(0, a, \Lambda)=0
$$

Again, once $\Psi, \Psi_{a}$ and $\Psi_{\Lambda}$ are known, Eq. (96) is a linear equation for $\Psi_{a \Lambda}$. Here the source term is nonzero, while the initial conditions (97) and (98) are zero. Substituting in Eqs. (94)- 98 $a=a_{c}$ and $\Lambda=\Lambda_{c}$, we obtain the second and fourth rows of Table 1 The other rows of Table 1 can be obtained in a similar way.

The functions $\Psi^{\prime}\left(x, a_{c}, \Lambda_{c}\right)$ and $\Psi_{a}\left(x, a_{c}, \Lambda_{c}\right)$ are special, as they satisfy the homogeneous form of Eq. (93): $S(x)=0$. Therefore, once they are found, the rest of the functions from Table 1 can be expressed through them:

$$
w(x)=\frac{1}{\Lambda_{c} Q\left(a_{c}\right)}\left[\Psi^{\prime}(x) \int_{0}^{x} \Psi_{a}(\xi) S(\xi) d \xi-\right.
$$

$$
\left.-\Psi_{a}(x) \int_{0}^{x} \Psi^{\prime}(\xi) S(\xi) d \xi\right]+C_{1} \Psi_{a}(x)+C_{2} \Psi^{\prime}(x),
$$

where $C_{1}$ and $C_{2}$ are integration constants. To satisfy the zero initial conditions at $x=0$, we must choose $C_{1}=C_{2}=0$ in all cases. Furthermore, evaluating $w(x)$ at the thermal wall $x=1$, we observe that the term proportional to $\Psi_{a}(x)$ in Eq. (99) vanishes, as $\Psi_{a}(1)=0$ at the critical point. Therefore, for all functions from Table 1 , except $\Psi^{\prime}$ and $\Psi_{a}$, we obtain

$$
w\left(1, a_{c}, \Lambda_{c}\right)=\frac{\Psi^{\prime}(1)}{\Lambda_{c} Q\left(a_{c}\right)} \int_{0}^{1} \Psi_{a}(\xi) S(\xi) d \xi .
$$

[1] Granular Gases, edited by T. Pöschel and S. Luding (Springer, Berlin, 2001); Granular Gas Dynamics, edited by T. Pöschel and N. Brilliantov (Springer, Berlin, 2003); I. Goldhirsch, Annu. Rev. Fluid Mech. 35, 267 (2003). 
[2] E.L. Grossman, T. Zhou, and E. Ben-Naim, Phys. Rev. E 55, 4200 (1997).

[3] A. Kudrolli, M. Wolpert, and J.P. Gollub, Phys. Rev. Lett. 78, 1383 (1997).

[4] E. Livne, B. Meerson, and P.V. Sasorov, Phys. Rev. E 65, 021302 (2002).

[5] J.J. Brey, M.J. Ruiz-Montero, F. Moreno, and R. GarciaRojo, Phys. Rev. E 65, 061302 (2002).

[6] E. Khain and B. Meerson, Phys. Rev. E 66, 021306 (2002).

[7] M. Argentina, M.G. Clerc, and R. Soto, Phys. Rev. Lett. 89, 044301 (2002).

[8] E. Livne, B. Meerson, and P.V. Sasorov, Phys. Rev. E 66, 050301(R) (2002).

[9] B. Meerson, T. Pöschel, P.V. Sasorov, and T. Schwager, Phys. Rev. E 69, 021302 (2004); cond-mat/0208286

[10] R. Soto, M. Argentina, and M.G. Clerc, in Granular Gas Dynamics, edited by T. Pöschel and N. Brilliantov (Springer, Berlin, 2003), p. 317.
[11] J.T. Jenkins and M.W. Richman, Phys. Fluids 28, 3485 (1985).

[12] P. Résibois and M. De Leener, Classical Kinetic Theory of Fluids (Wiley, New York, 1977).

[13] E. Khain, B. Meerson, and P.V. Sasorov (unpublished).

[14] J.J. Brey, J.W. Dufty, C.S. Kim and A. Santos, Phys. Rev. E 58, 4638 (1998).

[15] H.B. Keller and D.S. Cohen, J. Math. Mech. 16, 1361 (1967).

[16] L.D. Landau and E. M. Lifshitz, Electrodynamics of Continuous Media (Pergamon Press, Oxford, 1984).

[17] K. Huang, Statistical Mechanics (Wiley, New York, 1987), p. 43.

[18] S. Chapman and T.G. Cowling, The Mathematical Theory of Non-Uniform Gases (Cambridge Univ. Press, Cambridge, 1990), p. 101.

[19] E.M. Lifshitz and L.P. Pitaevskii, Statistical Mechanics, Part 2 (Pergamon Press, Oxford, 1980). 